\title{
Identifying Genetic Variants in Adolescents With Oppositional Defiant Disorders and/or Conduct Disorders: A Brief Report
}

\author{
Ukamaka Marian Oruche PhD, RN, PMHCNS-BC, \\ Sydney E. Ross MS, \\ Janet S. Carpenter PhD, RN, FAAN, \\ Jamie Renbarger MD, MS
}

\begin{abstract}
PROBLEM: To add to diversity in our state biobank, we explored the feasibility of collecting genetic material from adolescents with Oppositional Defiant Disorder (ODD) and/or Conduct Disorder (CD) and their family members. We also preliminarily explored genetic factors associated with ODD and/or CD by comparing participant data to 1000 Genome Project data on minor allele frequencies.

METHODS: Adolescents with ODD and/or CD and family members provided saliva samples for genetic testing. We evaluated five single nucleotide polymorphisms (SNPs), respectively in the dopamine receptor subtype D2, dopamine receptor subtype D3, dopamine beta-hydroxylase, dopamine transporter gene SLC6A3, and alpha-2-adrenergic receptor genes. Fisher's exact tests were used to examine differences in minor allele frequencies for each SNP.

FINDINGS: 31 viable samples were genotyped from 15 affected adolescents and 16 unaffected family members; the $60 \%$ consent rate reflected high feasibility. Compared to the 1000 Genome Project frequencies, affected adolescents had higher frequencies of the genetic variant in the dopamine receptor subtype D2 $(p=0.05)$ and dopamine beta-hydroxylase $(p=0.03)$, but not of the other three SNPs examined.
\end{abstract}

This is the author's manuscript of the article published in final edited form as:

Oruche, U. M., Ross, S. E., Carpenter, J. S., \& Renbarger, J. (2016). Identifying Genetic Variants in Adolescents With Oppositional Defiant Disorders and/or Conduct Disorders: A Brief Report. Journal of Child and Adolescent Psychiatric Nursing, 29(3), 154-157. https://doi.org/10.1111/jcap.12153 
CONCLUSIONS: Collecting genetic materials from an ethnically diverse sample of affected adolescents and their families is feasible. We offer practical suggestions to strengthen the integrity of future research studies.

Key words: precision medicine, genetic variants, saliva samples

As a psychiatric mental health nurse in child and adolescent nursing, imagine having a tool that helps pinpoint your patients' diagnoses and the gene variants associated with their phenotypic or symptom presentations (Beachy, Olson, \& Berger, 2015). That is the promise that precision medicine or precision health holds for all Americans. But this promise will never be realized without large data sets of biological or genetic information from large cohorts of volunteer patients and individuals (Collins, 2015; National Institutes of Health [NIH], 2015), including those with mental health disorders who are diverse in age, gender, race, ethnicity, and other characteristics.

To add to the diversity in our state biobank, we explored the feasibility of collecting genetic material from adolescents with Oppositional Defiant Disorders (ODD) and/or Conduct Disorder (CD) (American Psychiatric Association [APA], 2013) and their family members. We added a sample collection arm to a small descriptive study focused on understanding needs of families of adolescents with ODD and/or CD. Since we had data on hand, albeit a small amount, we also explored genetic factors associated with ODD and/or CD by comparing saliva samples of affected adolescents and unaffected family members (e.g., parents, siblings) to the 1000 Genome Project minor allele frequencies (Durbin et al., 2010). The minor allele frequency is the frequency with which the least common allele occurs in a given population. This least common 
allele is known as the variant, and though expressing the variant may have no downstream effects, it can also cause a variety of phenotypic changes. These changes could include increasing the risk of developing a particular disorder, affecting the severity with which the disorder occurs, or altering the rate of metabolism of a specific medication.

Genetic variants are important to ODD and/or CD. Single nucleotide polymorphisms (SNPs) are a common type of genetic variation that occur when there is a change in a single nucleotide of the deoxyribonucleic acid (DNA) sequence. DNA is the genetic material inherited from parents. Studies, including seminal works by Comings and colleagues $(1996 ; 2000 a$; 2000b), have identified five SNPs relevant to persons with ODD and/or CD (Beaver et al., 2007; Kopecková et al., 2008; Lee et al., 2007). Four of these SNPs are in the dopaminergic gene family: dopamine receptor subtype D2 (DRD2, rs1800497), dopamine receptor subtype D3 (DRD3, rs6280), dopamine beta-hydroxylase ( $D B H$, rs1108580), and the dopamine transporter (DAT1) gene SLC6A3 (rs27072). The fifth SNP is for the alpha-2-adrenergic receptor gene (ADRA2A, rs18005544). These genes are also thought to be involved in the etiology of Attention Deficit Hyperactivity Disorder, which often co-occurs with ODD and/or CD (Johnson, 2015).

\section{Methods}

This focused genetic association study was part of a descriptive study of 52 individuals from 15 families of adolescents with ODD and/or CD that has been described in detail elsewhere (Oruche et al., 2014). All study protocols were approved by the university institutional review board. We obtained parental consent for themselves and their children as well as children's assents to obtain $10 \mathrm{ml}$ saliva samples to obtain DNA for future investigation such as: (1) determining how the body breaks down medicines, how medicines are carried through the body, or how well they work in adolescents with ODD and/or CD; and (2) evaluating inherited risk of 
developing emotional problems in family members (i.e., siblings and parents) of affected adolescents. We also explained that the DNA would not be used for anything other than the specified uses without the participants' written agreement. Participants received no compensation for the optional genomic study.

\section{Sample Collection and Processing}

For each subject, the $10 \mathrm{ml}$ saliva sample was collected into an Oragene $\mathbb{R} \bullet \mathrm{DNA}$ saliva collection cup (DNA Genotek Inc., Kanata, Ontario, Canada). Research staff sealed the cup to prevent evaporation and labeled it with the participant identification number. The samples were transported to the laboratory for storage and processing. DNA was isolated from the saliva samples using the manufacturer-supplied protocol (DNA Genotek Inc, 2015). The concentration of double-stranded DNA was quantified using the reagents and protocol for the Quant-iT Broad Range Assay Kit and the Qubit Fluorometer (Invitrogen, Carlsbad, California). The DNA samples were then transferred into de-identified cryovials and stored at $-80^{\circ} \mathrm{C}$ in secure freezers until genotyping was performed.

SNP genotyping was performed using all reagents and predesigned primers available from Life Technologies (Thermo Fisher Scientific Inc., Waltham, Massachusetts) in combination with Taqman Real-Time polymerase chain reaction assays. $5 \mu \mathrm{L}$ of $2 \mathrm{ng} / \mu \mathrm{L}$ DNA for each sample was added to each SNP primer and Taqman Genotyping Master Mix as directed by the manufacturer's published methods. No-template negative controls and a $10 \%$ quality control sampling were run on each plate. The polymerase chain reactions were run on a QuantStudio 12K Flex RT-PCR System by Life Technologies/Thermo Fisher Scientific Inc. 


\section{Statistical Analysis}

Feasibility was evaluated based on consent rates. Fisher's exact tests were used to evaluate differences in minor allele frequencies of each tested SNP in the affected adolescents' samples and the family samples compared to the 1000 Genome Project. A $p$-value of $\leq 0.05$ was considered significant. Results were not corrected for multiple comparisons in this pilot analysis. Analyses were performed in the statistical software R version 3.1.1.

\section{Results}

Of the 52 possible participants, 31 viable samples were genotyped: 15 from affected adolescents and 16 from unaffected family members. This consent rate of $60 \%$ was sufficient to judge the process of obtaining saliva in this population feasible. Adolescents were a mean age of $13.7 \pm 1.8$ years, mostly male (66.7\%), and mostly multiracial (46.7\%) or African American (33.3\%). Family members were biological or blood relatives including parents, grandparents, and siblings. They were a mean age of $36.8 \pm 15.9$ years old, mostly female $(75 \%)$, and African American (62.5\%) or Caucasian (32.3\%). Clinical diagnoses are shown in Table 1.

As shown in Table 2, adolescents with ODD and/or CD had significantly higher minor allele frequencies for SNPs in DRD2 and DBH compared to the 1000 Genome Project sample. Family members' minor allele frequency expression did not significantly differ from those in the 1000 Genome Project.

\section{Discussion}

Successful gathering of the genomic data in this project required collaboration between mental health researchers and genomic experts. We showed the feasibility of collecting genetic materials from an ethnically diverse sample of adolescents with ODD and/or CD and their families. Our findings reinforce the seminal work by Comings and his colleagues (2000a; 2000b) 
focused on the association of the DRD2 and DBH gene variants in children with ODD and/or CD. Our study is important in its inclusion of a relatively large sample of African American and multiracial adolescents with mental disorders and their family members. Inclusion of diverse populations is significant because we want to make sure that all persons, even those considered relatively vulnerable or with mental disorders, have the opportunity to contribute and benefit from genome-wide association studies (NIH, 2015; Oruche, Carpenter, Renbarger, \& Ross, 2016).

Although our feasibility study was limited by its small sample size, we learned valuable lessons and offer practical suggestions to strengthen the integrity of future research studies in three areas. First, in regard to informed consent, we anticipated that some participants would opt not to participate in the optional genomic study. But we did not clearly address their concerns regarding the privacy and security of data (Oruche et al., 2016). Guidelines for consenting are now available for researchers and should be used in the future (NIH, 2015). Researchers need to provide written and verbal information about privacy and confidentiality, research questions to be explored, and participants' access to the results if they wish to use them in informing their own treatment choices in the future.

Second, we encountered the common challenge in genomic studies of the validity and reliability of phenotypic data. For example, medical records documenting mental or psychiatric diagnoses would have been more valid and reliable than self-reported diagnoses. Family members of adolescents with ODD and/or CD have a high probability of carrying the gene in question and having no symptoms. For our study, adolescents' diagnoses were confirmed by medical records but family members self-reported their psychiatric diagnoses. This means that some family members may have been affected with ODD and/or CD. Likewise, the 1000 
Genome Project samples are not associated with phenotypic data so some persons in these samples could have had ODD and/or CD. In sum, phenotypic data are critically important in genomic studies for confidence and meaningful interpretation of study findings. Future research must collect phenotype data, ideally using standardized diagnostic tools.

Third, we lost some saliva samples because of inadequate quantity, the saliva did not mix well with the reagents, or lids of the collection cup were not tightly sealed and so the sample dried up. Proper training for research staff and clear instructions for volunteer participants are critical to collecting viable samples for data analyses.

In conclusion, our hope is that this brief report will serve as encouragement to other psychiatric-mental health nurses to assist with the collection, analysis, and interpretation of biological data, which will contribute to realizing the promise of precision health care. 


\section{References}

American Psychiatric Association. (2013). Diagnostic and statistical manual of mental disorders (5th ed.). Arlington, VA: American Psychological Association.

Beachy, S. H., Olson, S., \& Berger, A. C. (2015). Genomics-enabled learning health care systems: Gathering and using genomic information to improve patient care and research Workshop Summary. Roundtable on Translating Genomic-Based Research for Health: Board on Health Sciences Policy: Institute of Medicine. The National Academies Press, ISBN 978-0-309-37112-4. Retrieved from http://www.nap.edu/catalog/21707/genomics-enabled-learning-health-care-systemsgathering-and-using-genomic.

Collins, F., \& Varmus, H. (2015). A new initiative on precision medicine. The New England Journal of Medicine, 372(9), 793-795.

Comings, D. E., Wu, S., Chiu, C., Ring, R. H., Gade, R., Ahn, C., .. Muhleman, D. (1996). Polygenic inheritance of Tourette syndrome, stuttering, attention deficit hyperactivity, conduct, and oppositional defiant disorder: the additive and subtractive effect of the three dopaminergic genes--DRD2, D beta H, and DAT1. American Journal of Medical Genetics, 67(3), 264-88.

Comings, D. E., Gade-Andavolu, R., Gonzalez, N., Wu, S., Muhleman, D., Blake, H., ... MacMurray, J. P. (2000a). Comparison of the role of dopamine, serotonin, and noradrenaline genes in ADHD, ODD and conduct disorder: multivariate regression analysis of 20 genes. Clinical Genetics, 57(3), 178-96. 
Comings, D. E., Gade-Andavolu, R., Gonzalez, N., Wu, S., Muhleman, D., Blake, H., ... MacMurray, J. P. (2000b). Multivariate analysis of associations of 42 genes in ADHD, ODD and conduct disorder. Clinical Genetics, 58(1), 31-40.

DNA Genotek Inc. (2015). Laboratory protocol for manual purification of DNA from $0.5 \mathrm{~mL}$ of sample. 11. Retrieved from http://www.dnagenotek.com/US/pdf/PD-PR-006.pdf

Durbin, R. M., Abecasis, G. R., Altshuler, R. M., Auton, G. A. R., Brooks, D. R.;, Durbin, A.... Reid, J. (2010). A map of human genome variation from population-scale sequencing. Nature, 467(7319), 1061-1073. doi:10.1038/nature09534. PMC 3042601. PMID 20981092.

Johnson, A. C. (2015). Developmental pathways to attention-deficit/hyperactivity disorder and disruptive behavior disorders: Investigating the impact of the stress response on executive functioning. Clinical Psychology Review, 36C, 1-12.

Kopecková, M., Paclt, I., Petrásek, J., Pacltová, D., Malíková, M., \& Zagatová, V. (2008). Some ADHD polymorphisms (in genes DAT1, DRD2, DRD3, DBH, 5-HTT) in case-control study of 100 subjects 6-10 age. Neuro Endocrinology Letters, (2), 246-51.

Lee, S. S., Lahey, B. B., Waldman, I., Van Hulle, C, A., Rathhouz, P., Pelham, W, E., ... Cook, E. H. (2007). Association of Dopamine Transporter Genotype with Disruptive Behavior Disorders in an eight-year longitudinal study of children and adolescents. American Journal of Medical Genetics Part B (Neuropsychiatric Genetics), 144b, 310-317.

Oruche, U. M., \& Carpenter, J., S. (online publication ahead of print, 5/26/2016). Raising the level of nursing involvement in the national precision medicine initiative: an example. Journal of Child and Adolescent Psychiatric Nursing. 
Oruche, U.M., Draucker, C., Al-Khattab, H., Knopf, A., \& Mazurczyk, J. (2014). Interventions for family members of adolescents with disruptive behavior disorders. Journal of Child and Adolescent Psychiatric Nursing, 27(3), 99-108. doi: 10.1111/jcap.12078. PMCID: PMC4199581 